\title{
Constitutive Activation Drives Compartment-Selective Endocytosis and Axonal Targeting of Type 1 Cannabinoid Receptors
}

\author{
Christophe Leterrier, ${ }^{1}$ Jeanne Lainé, ${ }^{2}$ Michèle Darmon, ${ }^{3}$ Hélène Boudin, ${ }^{4}$ Jean Rossier, ${ }^{1}$ and Zsolt Lenkei ${ }^{1}$ \\ ${ }^{1}$ Laboratoire Neurobiologie et Diversité Cellulaire, Ecole Supérieure de Physique et de Chimie Industrielles-Centre National de la Recherche Scientifique, \\ Unité Mixte de Recherche 7637, 75013 Paris, France, ${ }^{2}$ Laboratoire de Neurobiologie du Cervelet, Université Pierre et Marie Curie Paris 6, Faculté de \\ Médecine Pitié Salpêtrière, 75013 Paris, France, ${ }^{3}$ Neuropsychopharmacologie, Université Pierre et Marie Curie-Institut National de la Santé et de la \\ Recherche Médicale (INSERM), Unité Mixte de Recherche 677, Faculté de Médecine Pitié Salpêtrière, 75013 Paris, France, and ${ }^{4}$ Equipe Mixte INSERM \\ 0350, Hôpital Saint Antoine, 75012 Paris, France
}

The type 1 cannabinoid receptor (CB1R) is one of the most abundant G-protein-coupled receptors (GPCRs) in the brain, predominantly localized to axons of GABAergic neurons. Like several other neuronal GPCRs, CB1R displays significant in vitro constitutive activity (i.e., spontaneous activation in the absence of ligand). However, a clear biological role for constitutive GPCR activity is still lacking. This question was addressed by studying the consequences of constitutive activation on the intracellular trafficking of endogenous or transfected CB1Rs in cultured hippocampal neurons using optical and electron microscopy. We found that constitutive activity results in a permanent cycle of endocytosis and recycling, which is restricted to the somatodendritic compartment. Thus, CB1Rs are continuously removed by endocytosis from the plasma membrane in the somatodendritic compartment but not in axons, where CB1Rs accumulate on surface. Blocking constitutive activity by short-term incubation with inverse agonist 1-(2,4-dichlorophenyl)-5-(4-iodophenyl)-4-methyl$\mathrm{N}$-4-morpholinyl-1 $\mathrm{H}$-pyrazole-3-carboxamide (AM281) results in sequestration of recycled CB1Rs on the somatodendritic plasma membrane. Long-term inhibition of endocytosis by cotransfection of dominant-negative proteins results in impaired axonal polarization of surface-bound CB1Rs. Kinetic analysis shows that the majority of newly synthesized CB1Rs arrive first to the somatodendritic plasma membrane, from where they are rapidly removed by AM281-sensitive constitutive endocytosis before being delivered to axons. Thus, constitutive-activity driven somatodendritic endocytosis is required for the proper axonal targeting of CB1R, representing a novel, conformation-dependent targeting mechanism for axonal GPCRs.

Key words: constitutive activity; inverse agonist; targeting; plasma membrane; GPCR; axon

\section{Introduction}

G-protein-coupled receptors (GPCRs) are membrane-spanning transducers that change conformation after activation, leading to mobilization of intracellular signaling pathways. GPCRs regulate important physiological functions and represent major therapeutic targets (Pierce et al., 2002). The term "receptor" reflects the traditional view that ligand binding is mandatory for the

Received Sept. 15, 2005; revised Jan. 27, 2006; accepted Jan. 28, 2006.

We thank Nathalie Sauvonnet and Alice Dautry-Varsat (Institut Pasteur, Paris, France), Robert Lodge (Institut National de la Recherche Scientifique-Institut Armand Frappier, Laval, Quebec, Canada), Hong Cao and David McNiven (Mayo Clinic, Rochester, NY), and Thomas Kirschhausen (Harvard Medical School, Boston, MA) for donating plasmids. We thank Michel Bornens and Manuel Thery (Institut Curie, Paris, France) for their help and assistance on optical imaging; Sébastien Vaie, an undergraduate student in our team, who extended the NeuronJ plugin; and Damien Carrel and Boris Emerit (Faculté de Médecine Pitié Salpêtrière, Paris, France) for their assistance with neuronal culture.

Correspondence should be addressed to Dr. Zsolt Lenkei, Laboratoire Neurobiologie et Diversité Cellulaire, Ecole Supérieure de Physique et de Chimie Industrielles-Centre National de la Recherche Scientifique Unité Mixte de Recherche 7637, 10 rue Vauquelin, 75231 Paris, Cedex 05, France. E-mail: zsolt.lenkei@espci.fr.

H. Boudin's present address: Institut de Transplantation et de Recherche en Transplantation, Institut National de la Santé et de la Recherche Médicale U.643, CHU Hôtel Dieu, Nantes, France.

DOI:10.1523/JNEUROSCI.5437-05.2006

Copyright $\odot 2006$ Society for Neuroscience $\quad$ 0270-6474/06/263141-13\$15.00/0 activation of these proteins. However, a considerable number of GPCRs, either wild-type or pathologically relevant mutants, display significant constitutive activity both in vitro and in vivo (de Ligt et al., 2000; Morisset et al., 2000): the equilibrium between inactive and active receptor conformations is shifted toward activation even in the absence of agonist ligands, leading to measurable basal activation of intracellular signaling pathways (Milligan, 2003). Antagonist ligands that inhibit this basal activation by stabilizing inactive receptor conformations are termed inverse agonists (Milligan and Bond, 1997), whereas true "neutral" antagonists inhibit binding of both agonists and inverse agonists without favoring the equilibrium between active or inactive conformations (Kenakin, 2004). Interesting consequences of constitutive activity were reported recently for GPCRs such as the type 1 cannabinoid receptor (CB1R), the $5-\mathrm{HT}_{2 \mathrm{C}}$ serotonin receptor, or the $\alpha_{1 \text { a }}$ adrenergic receptor (Leterrier et al., 2004; Marion et al., 2004; Morris et al., 2004). These constitutively active GPCRs are constitutively endocytosed from the plasma membrane and, thus, exhibit a mostly intracellular (endosomal) localization at steady-state. Inverse agonists inhibit this constitutive endocytosis, resulting in sequestration of recycled receptors on the plasma 
membrane, a process termed externalization (Miserey-Lenkei et al., 2002). However, the physiological relevance of the constitutive endocytic cycle and, more generally, the possible biological role(s) of constitutive activity for neuronal GPCRs remain mostly unknown.

Discovered as the neuronal receptor for $\Delta^{9}$-tetrahydrocannabinol (Matsuda et al., 1990), the major psychoactive substance of marijuana, CB1R represents one of the most abundant GPCRs in the nervous system, highly expressed in the cerebral cortex, hippocampus, basal ganglia, and cerebellum (Howlett et al., 2002). CB1R displays a high level of constitutive activity when heterologously expressed in either non-neuronal cells or neurons (Pertwee, 2005), and we have recently reported that this constitutive activation results in constitutive endocytosis and recycling of CB1R in transfected human embryonic kidney 293 (HEK293) cells (Leterrier et al., 2004). In the present study, we investigate the physiological relevance of these findings in neurons.

First, we examined distribution and trafficking of endogenous CB1Rs in cultured hippocampal neurons. Then, by using a tagged CB1R construct, we simultaneously discriminated CB1Rs in somatodendritic versus axonal compartments as well as surfacelocalized versus intracellular CB1Rs in a single neuron. We found that CB1R undergoes rapid constitutive activity-driven endocytosis from the somatodendritic plasma membrane, whereas CB1Rs on the axon are endocytosed very slowly and are localized on the plasma membrane under control conditions. Thus, the presence of constitutive activity-driven endocytosis in the somatodendritic compartment but not in the axonal compartment may be the driving force for $\mathrm{CB} 1 \mathrm{R}$ targeting to the axonal plasma membrane. Indeed, blockade of constitutive activity abolished the polarized surface distribution of newly synthesized CB1Rs. Based on these findings, we propose a new, conformation-driven targeting mechanism for axonal GPCRs.

\section{Materials and Methods}

Chemicals and antibodies. CB1R agonist $(R)-(+)-[2,3-$ dihydro-5methyl-3-(4-morpholinylmethyl)pyrrolo[1,2,3-de]-1,4-benzoxazin-6yl]-1-naphthalenylmethanone mesylate [WIN55,212-2 (WIN)] was from Sigma (St. Louis, MO), and CB1R inverse agonist 1-(2,4-dichlorophenyl)-5-(4-iodophenyl)-4-methyl- $N$-4-morpholinyl-1 $H$-pyrazole-3carboxamide (AM281) was from Tocris (Bristol, UK). Methyl- $\beta$ cyclodextrin $(\mathrm{MBCD})$ was from Sigma. All culture media and additives were from Invitrogen (San Diego, CA). Lipofectamine 2000 was from Invitrogen. The anti-CB1R C-terminal (C-Ter) and N-terminal (N-Ter) antibodies were produced by Eurogentec (Seraing, Belgium) and have been characterized previously (Leterrier et al., 2004). Monoclonal mouse anti-microtubule-associated protein 2 (clone HM-2) and monoclonal mouse anti-FLAG (clone M1) were from Sigma. Alexa-Fluor conjugated secondary antibodies were from Invitrogen. Fluorescent proteinencoding plasmids were from Clontech (Mountain View, CA).

Plasmids. Our validated CB1-enhanced green fluorescent protein (eGFP) construct (Leterrier et al., 2004) was used to produce the FLAGCB1 (FCB1)-eGFP and FCB1-enhanced yellow fluorescent protein (eYFP) constructs. CB1-eGFP plasmid was digested with BamHI and AgeI to excise the CB1R coding sequence, and inserted into eYFP-N1 plasmid. Both CB1-eGFP and CB1-eYFP were digested with SalI and Bam HI, and a synthetic oligonucleotide coding for an $\mathrm{NH}_{2}$-terminal cleavable signal sequence (MKTIIALSYIFCLVPA) followed by a FLAG epitope (DYKDDDDA) was inserted. Post-translational cleavage of the signal sequence allows proper expression of CB1R tagged with a free N-terminal FLAG epitope (Guan et al., 1992). The two resulting constructs, named FCB1-eGFP and FCB1-eYFP, were validated by comparing their steady state distribution to both CB1-wild type (WT) and CB1eGFP, and their agonist or inverse-agonist induced translocation in HEK293 cells (Leterrier et al., 2004). The Ras-related GTP-binding pro- tein ( $\mathrm{rab}$ )-enhanced cyan fluorescent protein (eCFP) plasmids were a generous gift from Dr. Robert Lodge (Université du Quebec, Montréal, Quebec, Canada). The light chain clathrin fused to red fluorescent DsRed protein (Engqvist-Goldstein et al., 2001) was a gift from T. Kirschhausen (Harvard Medical School, Boston, MA). The wild-type and mutant dynamins fused to eGFP plasmids (Cao et al., 1998) were gifts from Dr. Mark A. McNiven (Mayo Clinic, Rochester, MN). The dynamin cDNAs were extracted by digestion with EcoRI and HindIII, and inserted in eCFP-N1 plasmid. The wild-type and mutant eps15-eGFP plasmids were gifts from Dr. Alice Dautry-Varsat (Institut Pasteur, Paris, France) (Benmerah et al., 2000). The eGFP sequence was cut out by digesting the plasmids with BsrG1 and NheI, before insertion of the eCFP sequence obtained from digestion of the eCFP-C1 plasmid with the same enzymes.

Hippocampal neuronal cultures. Hippocampal neuronal cultures were performed essentially as described by Goslin et al. (1998) with some modifications (Jolimay et al., 2000). Briefly, hippocampi of rat embryos were dissected at embryonic days 18-19. After trypsinization, dissociation was achieved with a fire-polished Pasteur pipette. Cells were counted and plated on poly-L-lysine-coated 12-mm-diameter glass coverslips or 15-mm-diameter Thermanox coverslips (for electron microscopy), at a density of 300-400 cells $/ \mathrm{mm}^{2}$. The plating medium was Neurobasal supplemented with $2 \%$ B27 and containing Glutamax I (0.5 mM) and penicillin $\mathrm{G}(10 \mathrm{U} / \mathrm{ml}) /$ streptomycin $(10 \mu \mathrm{g} / \mathrm{ml})$. Four hours after plating, the coverslips were transferred into Petri dishes containing supplemented Neurobasal medium that had been conditioned for $24 \mathrm{~h}$ on a confluent glia layer.

Neurons were transfected after $8-10 \mathrm{~d}$ in vitro (DIV) using Lipofectamine 2000, following the manufacturer's instructions. They were treated and processed $24 \mathrm{~h}$ after transfection. For pharmacological treatments, concentrated ligands dissolved in dimethylsulfoxide (DMSO) were directly added to the culture medium. The highest final concentration reached was $0.2 \%$ DMSO, and control experiments with up to $0.5 \%$ DMSO have shown the absence of effects on neuronal morphology and on the cellular distribution of CB1Rs (data not shown).

Immunocytochemistry. For standard immunocytochemistry, neurons were briefly rinsed in with Dulbecco's PBS (DPBS; Invitrogen, catalog \#14040-083) and fixed in DPBS containing 4\% paraformaldehyde and $4 \%$ sucrose. After permeabilization with 5 min incubation in DPBS containing $0.1 \%$ Triton X-100 and block for $30 \mathrm{~min}$ in antibody buffer (DPBS supplemented with $2 \%$ BSA and 3\% normal goat serum), neurons were incubated with primary antibodies diluted 1:400-1:800 in antibody buffer for $1-2 \mathrm{~h}$ at room temperature or $4^{\circ} \mathrm{C}$ overnight. After DPBS rinses, neurons were labeled with secondary antibodies diluted to 1:400 in antibody buffer for $1 \mathrm{~h}$ at room temperature.

For live surface CB1R staining, anti-FLAG M1 antibody was added at 1:500-1:1000 to the culture medium and incubated for $5 \mathrm{~min}$ at $37^{\circ} \mathrm{C}$. Neurons were briefly rinsed with DPBS, fixed in DPBS containing $4 \%$ paraformaldehyde, $4 \%$ sucrose, and incubated for $45 \mathrm{~min}$ in Alexa-568 goat anti-mouse secondary antibody diluted to 1:400. Coverslips were finally mounted in Mowiol (Calbiochem, La Jolla, CA) eventually containing Hoechst 33432 diluted to 1:1000.

For antibody feeding experiments, neurons were incubated for $1 \mathrm{~h}$ with anti-FLAG M1 antibody (1:800) before fixation, permeabilization, and staining with goat anti-mouse Alexa-568 secondary antibody (1: 600). To strip M1 antibody bound to surface receptors, neurons were eventually put on ice at the end of antibody feeding and subjected to a 4 min incubation at $4^{\circ} \mathrm{C}$ with a solution of $0.5 \mathrm{M} \mathrm{NaCl}$ and $0.2 \mathrm{M}$ acetic acid, $\mathrm{pH} 3.5$, before being fixed.

BFA block. Brefeldin A (BFA) was used to accumulate synthesized CB1Rs in a golgi-endoplasmic reticulum (ER) intracellular compartment, and to allow a pulse of receptor transport after BFA washout (Wisco et al., 2003; Fache et al., 2004). Neurons were transfected as described above with FCB1-eGFP. After $3 \mathrm{~h}$, the transfection medium was removed and BFA was added to $0.5-0.75 \mu \mathrm{g} / \mathrm{ml}$ of final concentration. After $12-18 \mathrm{~h}$, coverslips were washed twice in conditioned medium and transferred to fresh dishes containing conditioned medium, either with vehicle $(0.2 \%$ DMSO) or AM281 $(10 \mu \mathrm{M})$. Coverslips were removed and fixed at indicated times and stained for surface CB1R with antiFLAG M1 antibody as described above. All transfected neurons for each 
condition were classified based on the surface CB1R distribution (supplemental Fig. 4, available at www.jneurosci.org as supplemental material). Classes were "not yet on surface" (CB1R is expressed as seen in the eGFP channel but still retained in ER/golgi and no surface labeling is detectable), "uniform" (CB1R is present on both somatodendritic and axonal surface), "axonal" (surface CB1R distribution is polarized mostly to the axonal plasma membrane, similar to untreated neurons), and "somatodendritic" (reversed polarization of CB1R distribution, most surface CB1R being on the surface of soma and dendrites). Proportion of neurons in the different classes was calculated in respect to the total number of transfected neurons per coverslip (ranging from 50 to 150 for each condition, the experiment being done in two independent replicates).

Microscopy. Confocal images were taken on a Leica (Nussloch, Germany) TCS NT confocal laser-scanning microscope by the use of dry $20 \times$, numerical aperture (NA) 0.7 , oil immersion $40 \times$, NA 1.2, and oil immersion $63 \times$, NA 1.32 objectives. Widefield images were taken on a Leica DM-R or a Leica DM-IRB using dry $20 \times$, NA 0.7 , oil immersion $40 \times$, NA 1.2, and oil-immersion 100×, NA 1.4 objectives and Coolsnap HQ (Roper Scientific, Trenton, NJ), QImaging (Burnaby, British Columbia, Canada) QICAM or Coolsnap Fx camera, depending on experiments. In all cases, emission and excitation filters proper to each fluorophore were used sequentially and the absence of cross talk between different channels was checked with selectively labeled preparations.

For quantification of the surface/total ratio (S/T ratio), $63 \times$ single confo$\mathrm{cal}$ images through the somatodendritic compartment were acquired. Alternatively, $100 \times$ widefield images were used in another set of experiments. Images from the green (total FCB1-eGFP or FCB1-eYFP fluorescence) and red (surface CB1R labeling) were used for quantification.

For quantification of the axon/dendrites ratio (A/D ratio), $20 \times$ images of transfected neurons were used. The images from either the green channel (total CB1R) or the red channel (surface CB1R) were used for quantification.

For certain illustrative images, $40 \times$ images were stitched to visualize the axonal arborization and $100 \times$ images of somas were generated by imaging stacks of 40-60 images, $0.2 \mu \mathrm{m}$ apart, subsequently deconvoluted using Volocity 3 (Improvision, Lexington, MA). Calculated point spread functions (PSFs) for the different wavelengths were used assuming a $100 \times$, NA 1.1 objective after measurement of the real objective NA obtained from experimental PSFs obtained by imaging $0.2 \mu \mathrm{m}$ Tetraspeck beads (Invitrogen). The iterative maximum entropy algorithm was set to $32-35$ iterations.

Preembedding immunocytochemistry and electron microscopy. All procedures were applied to the neurons in situ (i.e., directly on their culture substratum) (plastic Thermanox coverslips; Nunc, Rochester, NY). To obtain enhanced sensitivity, a two-step labeling protocol of neurons was performed by incubating for $1 \mathrm{~h}$ with an antibody directed against the extracellular $\mathrm{N}$ terminus of CB1R (N-Ter antibody) followed by fixation, permeabilization, and immunolabeling with an antibody directed against the intracellular $\mathrm{C}$ terminus of CB1R (C-Ter antibody). As both N-Ter and $\mathrm{C}$-Ter antibodies were produced in rabbit, the same secondary anti-rabbit serum was used to reveal both primary antibodies.

DIV 9 neurons were first incubated for $1 \mathrm{~h}$ at $37^{\circ} \mathrm{C}$ or room temperature with $\mathrm{N}-\mathrm{Ter}$ antibody diluted 1:400 in the culture medium, and then fixed 30 min with $4 \%$ paraformaldehyde, $0.1 \%$ glutaraldehyde, and $5 \%$ sucrose in $0.1 \mathrm{~m}$ phosphate buffer. Two aldehyde-quenching steps in $0.1 \%$ sodium borohydrate and $0.1 \mathrm{~m}$ glycine preceded the blocking step of nonspecific sites in 5\% normal goat serum diluted in PBS with $0.01 \%$ saponin. One hour of incubation was then performed with the C-Ter antibody (1:1000) in PBS with $0.01 \%$ saponin (step omitted for the $\mathrm{N}$-Ter antibody feeding experiment).

For immunoperoxidase procedures, a biotinylated anti-rabbit IgG (Vector Laboratories, Burlingame, CA) was applied as secondary antibody (1:200 in PBS plus $0.01 \%$ saponin), and the ABC peroxidase complex (Vectastain Elite; Vector Laboratories) was used for amplification. Revelation was performed by using $0.05 \%$ diaminobenzidin. For immunogold labeling, overnight incubation in ultra-small gold conjugate $\mathrm{F}\left(\mathrm{ab}^{\prime}\right)_{2}$ fragments of goat anti-rabbit IgG (1:100; Aurion, Wageningen,
The Netherlands) was followed by extensive washings, 10 min postfixation in $2 \%$ glutaraldehyde, and finally, a silver enhancement reaction (Aurion R-Gent SE-ME kit).

After $\mathrm{OsO}_{4}$ postfixation ( $2 \%$ for immunoperoxidase material and $0.5 \%$ for immunogold-labeled materials) and $2 \%$ uranyl acetate en bloc staining, immunostained cells plated on their coverslips were dehydrated in graded acetone and finally embedded in Durcupan resin (Fluka, Buchs, Switzerland). Light microscopic visualizations were done on a Leica DMR microscope equipped with $40 \times$ or $100 \times$ objectives and a Nikon (Tokyo, Japan) DXM 1200 digital camera. Ultrathin sections were examined with a CM100 electron microscope (Philips Electron Optics, Eindhoven, The Netherlands), operated at $60 \mathrm{kV}$.

Image quantification and analysis. Three quantification methods were devised and implemented either on ObjectImage (http://simon.bio. uva.nl/object-image.html) or ImageJ (http://rsb.info.nih.gov/ij/) software (see below). All measurements were plotted and analyzed using Graph Pad (San Diego, CA) Prism software. Significance of differences between various conditions was calculated using one-way ANOVA with Newman-Keuls post-tests for computing $p$ estimates. For significance symbols (asterisks or crosses), one symbol means $p<0.05$, two means $p<0.01$, and three means $p<0.001$.

For quantification of subneuronal CB1R localization, several sections of whole somas exhibiting CB1R-positive immunogold labeling were assembled from high-magnification EM images and gold beads were classified based on their ultra-structural localization as endosomal, intracellular nonendosomal, plasma membrane bound, or axonal (when a CB1R-positive axon passes along the neuronal soma). This was done for six neurons from two independent experiments and yielded the average subneuronal localizations shown in Figure $1 \mathrm{Ba}$.

For measurement of endogenous CB1R density along somatodendritic plasma membrane, EM images of whole somas (magnification, $4800-6500 \times$ ) from neurons labeled with N-Ter plus C-Ter antibodies and immunoperoxidase revelation were used. The plasma membrane of each soma was outlined using an assisted semiautomatic method (NeuronJ) originally developed by E. Meijering (Meijering et al., 2004) as a plugin for the ImageJ software, which detects ridge-like image features and generates optimized tracings along the centerline of ridges between user-defined key points. The plugin was modified to measure intensities along the tracings, thus allowing us to calculate the mean immunoperoxidase staining along the entire neuronal plasma membrane for a single section. This value was named the surface labeling (S). This was divided by the mean intensity along the outlined nuclear membrane $(\mathrm{N})$, which was used as a normalization measurement because it is devoid of CB1R labeling and takes into account differences of membrane labeling density caused by possible variation in experimental conditions (osmification) and imaging (photography and scanning). The surface/nucleus ratio ( $\mathrm{S} / \mathrm{N}$ ratio) is used in Figure $4 B$.

For quantification of the CB1R surface/total ratio, a single highresolution image of the cell body of 24-36 neurons from two to three independent experiments was used. The somatodendritic compartment was selected on the GFP (or YFP) image and the mean fluorescence of the GFP and the Alexa-568 channels in this region was measured. The ratio of these two mean fluorescences led to the S/T ratio as shown in Figure $5 B a, B b$.

For measurement of surface CB1R polarization, widefield $20 \times$ images of neurons were used. An approach similar to previous studies of protein exhibiting axonal distribution was used (Sampo et al., 2003; Wisco et al., 2003): segments were traced along morphologically identified axon and dendrites on the surface-labeled CB1R image, and the A/D ratio was computed from mean fluorescence along these segments. The A/D ratio could be calculated for eGFP images (green channel), thus measuring compartmentalization of the total CB1R population (see Fig. $5 B c$ ), or for surface CB1R images (red channel), thus measuring polarization of surface CB1R expression (see Fig. 6 F). An axonal protein has a $>1 \mathrm{~A} / \mathrm{D}$ ratio, whereas a somatodendritic distribution leads to a $\angle 1 \mathrm{~A} / \mathrm{D}$ ratio (Sampo et al., 2003). 


\section{Results \\ CB1R localization in cultured hippocampal neurons}

To study the subcellular localization and trafficking of CB1R, we used cultured hippocampal neurons after DIV 8-9, when polarization of axons and dendrites is complete (Dotti et al., 1988) and synapses have formed (Fletcher et al., 1994). Using a two-step labeling protocol against both the extracellular $\mathrm{N}$ terminus and intracellular $\mathrm{C}$ terminus of the receptor followed by immunoperoxidase revelation (see Materials and Methods), CB1Rs are detected in a subpopulation of neurons, representing $5-10 \%$ of the total cell population (Fig. $1 A$ ), all displaying immunoreactivity for the GABA-synthesizing enzyme GAD65 (65 $\mathrm{kDa}$ glutamic acid decarboxylase) (data not shown). In these neurons, CB1Rs are present in axons and in somatodendritic intracellular vesicles. Correlated electron microscopy confirmed that axonal CB1Rs are localized predominantly to the axonal surface, whereas somatodendritic CB1Rs are found mostly in large intracellular vesicles often showing complex, membranous intraendosomal morphology. A general characteristic of the CB1R-immunoreactive neurons is a relatively electrolucent cytoplasm with a well developed smooth ER and a large indented nucleus containing a prominent nucleolus. CB1R-positive "en passant" axons are often seen running along CB1R-positive somas, although it is not possible to determine which neuron a particular axon belongs to, because of high density and branching of CB1R-positive axons in the neuronal culture. Immunogold labeling of CB1Rpositive somas allowed us to estimate the proportion of CB1Rs in endosomes and on the plasma membrane in the somatodendritic compartment, with $\sim 30 \%$ of receptors localized to endosomes and between 10 and 20\% of receptors expressed on the plasma membrane, the rest being intracellular, nonendosomal receptors (Fig. $1 \mathrm{Ba}$ ). Interestingly, CB1Rs are uniformly distributed along the axonal plasma membrane and do not exhibit a punctate localization pattern, suggesting that they are not enriched in synaptic varicosities (Fig. $1 \mathrm{Bb}$ ), an observation compatible with recent in vivo data on CB1R distribution on axons of hippocampal interneurons (Nyiri et al., 2005). A similar distribution is detected in older, DIV 29 neurons that possess fully mature synapses (Fig. 1C). In conclusion, endogenous CB1Rs in cultured hippocampal neurons at DIV 8-9 are expressed predominantly on the axonal plasma membrane and in somatodendritic endosomes of GABAergic interneurons.

We then transfected low-density cultures of hippocampal neurons at DIV 8-10 with an epitope-tagged CB1R bearing eGFP attached to the $\mathrm{C}$ terminus and a FLAG tag at the end of the extracellular $\mathrm{N}$ terminus (FCB1-eGFP). Addition of the C-terminal eGFP tag does not disturb the signaling and trafficking characteristics of CB1R in transfected HEK293 cells (Leterrier

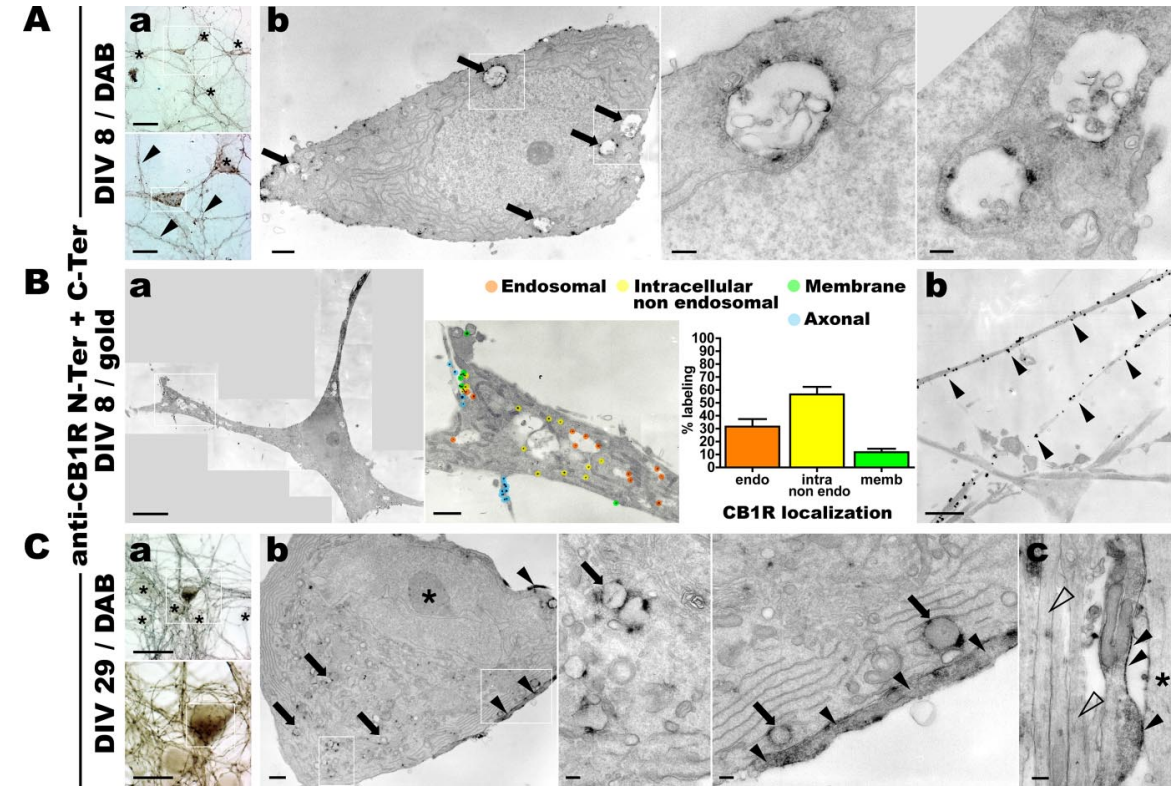

Figure 1. In cultured hippocampal neurons, endogenous CB1Rs localize at the axonal surface and in somatodendritic endo-

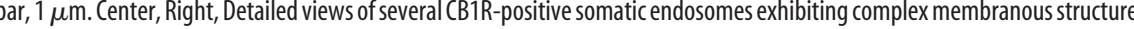
nded by immunonegative neurons (asterisks). Scale bars: top, $50 \mu \mathrm{m}$; bottom, $20 \mu \mathrm{m}$. Cb, Correlated electron 1R-positive axons passing along the soma (arrowheads). Left, Note the deeply indented nucleus with prominent nucleolus (asterisk). Scale bars: left, $1 \mu \mathrm{m}$; center, right, $0.2 \mu \mathrm{m}$. (c, Axonal bundle along a dendrite (asterisk) containing a CB1R-positive axon with homogenous labeling along the plasma membrane (arrowheads) and immunonegative axons (empty arrowheads). Scale bar, $0.2 \mu \mathrm{m}$.

et al., 2004), nor did addition of the small N-terminal FLAG (see Materials and Methods). In our hands, the expression level of FCB1-eGFP is comparable with that of endogenous CB1Rs, as shown by the comparable intensities of labeling with the C-Ter antibody (Fig. $2 \mathrm{~A}$, middle panel in red). The FCB1-eGFP receptor is properly targeted to axons and present in somatodendritic vesicles (Fig. $2 B$, boxed soma), similarly to the subcellular distribution of endogenous CB1Rs, as seen in a nontransfected CB1Rpositive neuron nearby (lower boxed soma; see also indirect comparison in supplemental Fig. $1 A, B$, available at www.jneurosci. org as supplemental material). Simultaneous detection of surface (extracellular FLAG epitope) and total receptor (eGFP) populations confirms that, similarly to endogenous CB1Rs, axonal FCB1-eGFPs are mostly surface-bound, whereas somatodendritic FCB1-eGFPs are predominantly intracellular (Fig. 2 B). A control experiment measuring the proportion of FCB1-eGFPs that are present on the somatodendritic plasma membrane of individual neurons displaying various expression levels ( $\mathrm{S} / \mathrm{T}$ ratio) (Fig. 2C), shows that endosomal localization does not depend on the total quantity of receptors expressed by a particular 


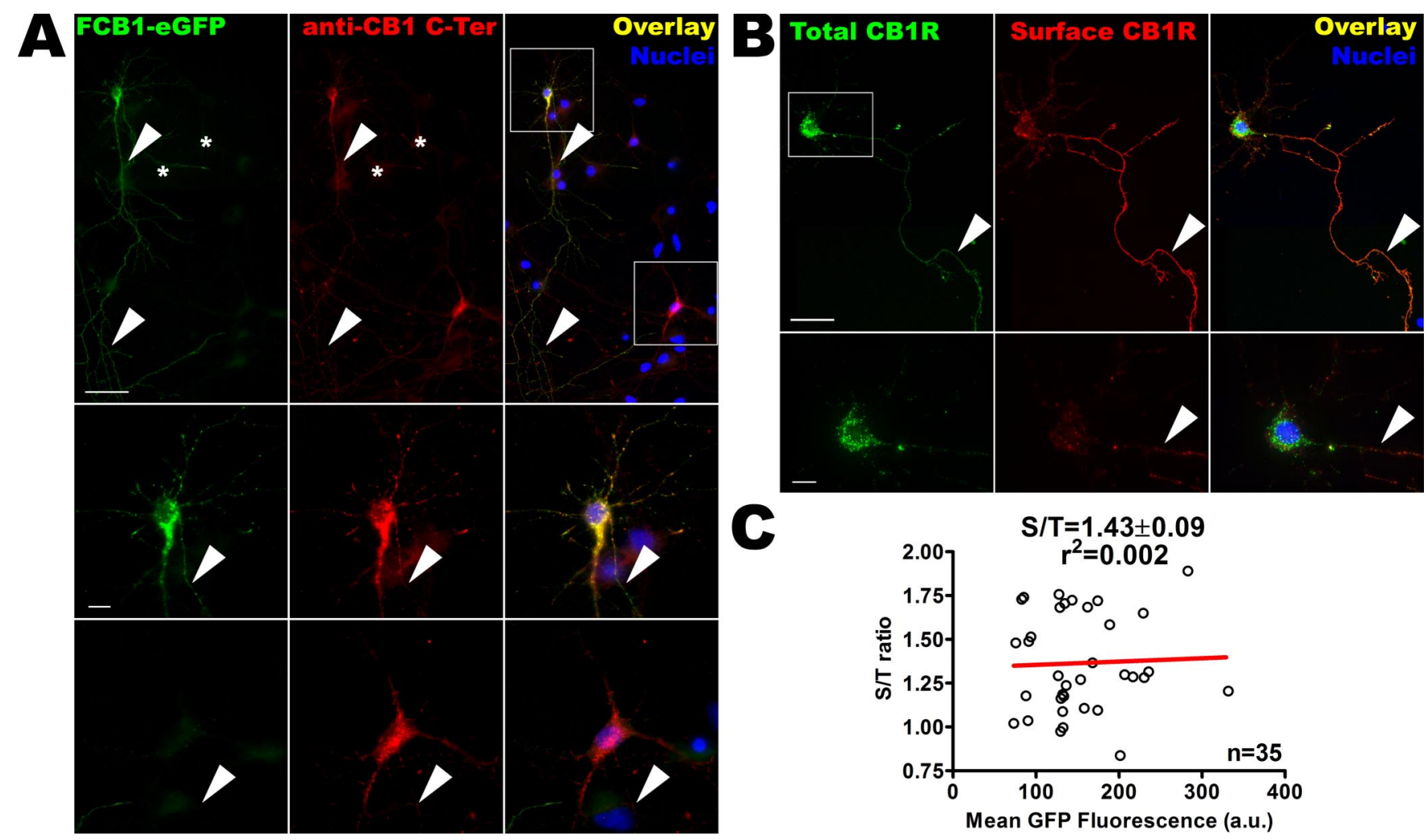

Figure 2. FCB1-eGFP is properly expressed and targeted to the axonal surface and somatodendritic endosomes. A, Comparison of the distribution of endogenous CB1R and transfected FCB1-eGFP by detection of eGFP fluorescence (in green) and labeling with the anti-CB1R C-Ter antibody (labeling both endogenous and transfected CB1Rs, red). The top shows soma, dendrites, and axonal arborization of an FCB1-eGFP transfected neuron (top left box) together with a neuron expressing endogenous (B1R (bottom right box). Positive neurons are surrounded by (B1-negative neurons (asterisks, nuclear staining in blue). CB1R as well as FCB1-eGFP show vesicular staining in the somatodendritic compartment (boxed) and homogenous staining on the axon (arrowheads). Notably, the comparable intensity of C-Ter antibody labeling (red) shows that transfection of FCB1-eGFP leads to expression levels similar to those of endogenous CB1Rs. Bottom panels show details of neurons boxed at the top. Vesicular staining can clearly be seen in the soma and in dendrites, together with emergence of the labeled axon (arrowheads). Scale bars: top, $50 \mu \mathrm{m}$; bottom panels, 10 $\mu \mathrm{m} . \boldsymbol{B}$, Presence of the FLAG epitope allows highly sensitive labeling of surface receptors (in red) by rapid live incubation of transfected neurons with M1 anti-FLAG antibody. Intracellular eGFP labeling (in green) is brighter than surface eGFP labeling, because of the concentration of receptors in endosomes. Because of the high sensitivity of the red FLAG epitope surface labeling, surface CB1Rs are far more visible in the red channel than in the green channel (see in axons where surface receptors are more visible in the red channel than in the green channel; arrowheads). Surface (B1Rs are hardly detectable on the somatodendritic plasma membrane [see red channel in boxed region, detailed in bottom image], whereas they are concentrated in the axon (arrowheads). Scale bars: top, $50 \mu \mathrm{m}$, bottom, $10 \mu \mathrm{m}$. C, FCB1-eGFP construct allows to measure the CB1R S/T ratio on the somatodendritic compartment of individual neurons using the ratio between green (eGFP total receptor fluorescence) and red (surface labeling with $\mathrm{M} 1$ antibody) channels. This ratio does not depend on the FCB1-eGFP level of expression in each particular neuron; there is no correlation between the value of mean eGFP fluorescence and the value of the S/T ratio for 35 individual somas of FCB1-eGFP expressing neurons in the control condition, as plotted here $\left(r^{2}<0.02\right.$, as assessed by Pearson's test). This implies that surface expression of (B1R does not depend on transfection potency or variation in expression levels between individual neurons or between experimental conditions. The data points shown come from three independent experiments.

neuron, as shown by the lack of correlation $\left(r^{2}=0.002\right.$, Pearson's test) between eGFP fluorescence and the corresponding $\mathrm{S} / \mathrm{T}$ ratio. Electron microscopy using anti-eGFP antibody confirmed the similar localization for endogenous and transfected CB1Rs, namely on the axonal surface and in large and complex somatodendritic vesicles (supplemental Fig. 1C, available at www. jneurosci.org as supplemental material). Finally, incubation of transfected neurons with protein synthesis inhibitor cycloheximide (10 $\mu \mathrm{g} / \mathrm{ml}$ ) for up to $6 \mathrm{~h}$ (Goslin and Banker, 1990) failed to induce significant reduction in the intracellular labeling of CB1R (data not shown), indicating that the majority of intracellular CB1Rs are not in the neosynthetic pathway, but could be rather of endocytic origin.

\section{$\mathrm{CB} 1 \mathrm{R}$ is constitutively endocytosed primarily in the somatodendritic compartment}

To test whether endosomal CB1Rs correspond to receptors constitutively endocytosed from the plasma membrane, we incubated live neurons with the N-Ter antibody directed against the extracellular $\mathrm{N}$ terminus of endogenous CB1R. After $2 \mathrm{~h}$, cells were fixed, permeabilized, and the N-Ter antibody was revealed with immunoperoxidase staining (Fig. 3A). The N-Ter antibody labeled surface receptors on axons but also numerous endosomes in the somatodendritic compartment, showing that receptors labeled with the N-Ter antibody have been constitutively endocytosed. At the EM level, recently endocytosed CB1Rs are localized to large endosomes displaying a complex membranous structure, often in close apposition to the somatodendritic plasma membrane (Fig. 3Ab). Strikingly, in axons, we did not observe intracellular CB1R-positive endosomes or pits, suggesting that constitutive endocytosis occur mostly from the somatodendritic surface.

These results were confirmed and extended using FCB1-eGFP transfected neurons that were incubated for $1 \mathrm{~h}$ with the M1 antibody directed against the extracellular FLAG epitope (Fig. $3 B, C)$. Detection of the M1 antibody with a red fluorescent secondary antibody results in intense labeling of the axonal surface. Importantly, surface labeling of the somatodendritic compartment is barely visible, whereas the majority of CB1R-containing somatodendritic vesicles also contained the M1 antibody (Fig. 
$3 B$, compare the punctate red labeling with surface labeling alone in Fig. $2 B$ ). Interestingly, M1 antibody incubation of neurons cotransfected with FCB1-eGFP and clathrin-DsRed (Engqvist-Goldstein et al., 2001) leads to colocalized puncta of eGFP, clathrin, and endocytosed M1 in the soma and dendrites of transfected neurons (supplemental Fig. 2, available at www. jneurosci.org as supplemental material), suggesting that CB1R constitutive endocytosis is primarily clathrin-mediated. To visualize more specifically endocytosed receptors, the M1 antibody bound to surface CB1Rs was stripped with an acid wash after feeding. Surface axonal labeling is greatly reduced, whereas soma and dendrites still contain brightly labeled puncta (Fig. 3C). This suggests that a substantial proportion of the intracellular CB1Rs found in the soma and dendrites are in fact of recent $(<1 \mathrm{~h})$ endocytic origin. The relative paucity of endosomal labeling in the axonal compartment suggest that axonal receptors mostly reside on the plasma membrane and are not subject to intense constitutive endocytosis. The M1 antiFLAG antibody feeding was CB1Rspecific, as shown by the lack of red labeling in nontransfected cells, and did not provoke $\mathrm{CB} 1 \mathrm{R}$ endocytosis by itself because the localization of FCB1-eGFP labeling (total CB1R population) is not changed whether M1 incubation is absent (supplemental Fig. $1 B$, available at www. jneurosci.org as supplemental material), short (Fig. 2 B, 5 min for surface labeling), or long (Fig. 3B, $1 \mathrm{~h}$ feeding; supplemental Fig. 2, available at www.jneurosci.org as supplemental material). In conclusion, antibody feeding experiments show that $\mathrm{CB} 1 \mathrm{R}$ is constitutively endocytosed in neurons, particularly in the somatodendritic compartment.

\section{Pharmacology and trafficking of CB1Rs in cultured hippocampal neurons}

We then asked whether the observed constitutive endocytosis of $\mathrm{CB} 1 \mathrm{R}$ is linked to the pharmacological state of the receptor. Using the highly sensitive preembedding two-step immunocytochemistry for electron microscopy, it was possible to detect and quantify endogenous CB1R expression in the somatodendritic compartment of cultured neurons, including the weak surface expression on the somatodendritic plasma membrane (Fig. 4A). To reveal the antibodies, immunoperoxidase revelation was used because immunogold labeling was not sensitive enough to yield consistent quantitative results. For the quantification of membrane labeling density, we developed a semiautomatic method based on the NeuronJ plugin (Meijering et al., 2004) that minimizes the impact of human intervention by automatically detecting the local maximum of labeling density. The mean labeling intensity along the plasma membrane $(\mathrm{S})$ was measured and the mean intensity along the nuclear envelope of the same cell $(\mathrm{N})$, devoid of CB1R staining, was used as an internal control of mem- brane labeling intensity ( $\mathrm{S} / \mathrm{N}$ ratio; see Materials and Methods) (Fig. $4 B$ ).

In vehicle-treated cells, similarly to nontreated neurons (see above), CB1Rs are present predominantly inside endosomes but also on discrete locations along the plasma membrane. CB1Rpositive en passant axons running along the somatic membrane are also clearly distinguishable. Neurons treated for $3 \mathrm{~h}$ with the agonist WIN ( $1 \mu \mathrm{M})$ exhibit more CB1R-positive endosomes and are devoid of surface labeling on the somatodendritic plasma membrane, except inside small pits. On labeled axons, CB1Rs are downregulated but are still present on the plasma membrane. After $3 \mathrm{~h}$ treatment with the inverse agonist AM281 (Gifford et al., 1997) at $10 \mu \mathrm{M}, \mathrm{CB} 1 \mathrm{Rs}$ are upregulated at the somatodendritic plasma membrane, localized to intensely stained patches and large pit-like structures, and are abundant on axons. Quantification of the immunoperoxidase staining intensity on the plasma membrane shows that somatodendritic surface receptors are significantly downregulated by WIN treatment, whereas inverse agonist AM281 leads to enhanced surface localization (Fig. 4B). This suggests that WIN promotes endocytosis from the plasma membrane, whereas AM281 inhibits constitutive endocytosis, re- 

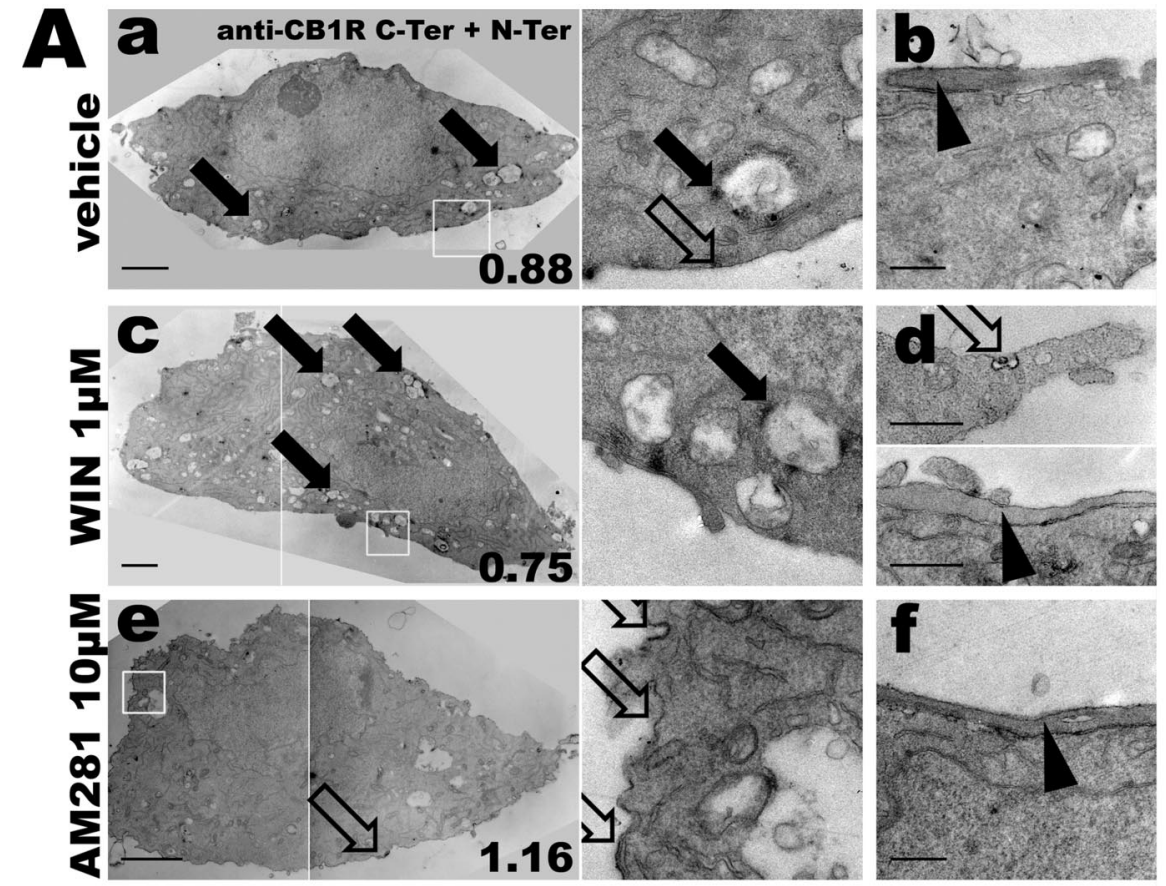

B

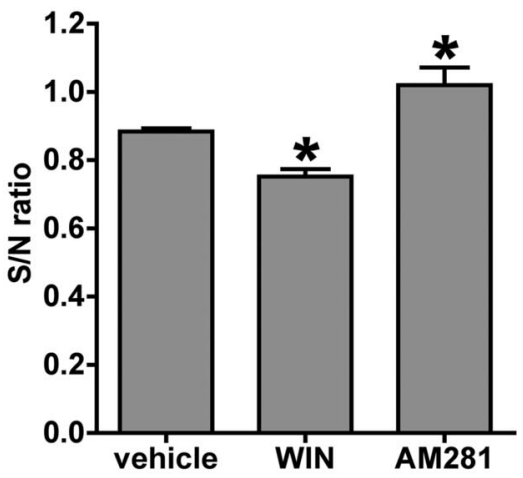

Figure 4. Somatodendritic trafficking of endogenous CB1R after pharmacological treatments. $\boldsymbol{A}$, Hippocampal neurons at DIV 9 are incubated for $3 \mathrm{~h}$ with either $0.2 \%$ DMSO as vehicle ( $\boldsymbol{A} \boldsymbol{a}, \boldsymbol{A} \boldsymbol{b}$ ), $1 \mu \mathrm{m}$ agonist WIN $(\boldsymbol{A c}, \boldsymbol{A d})$, or $10 \mu \mathrm{m}$ inverse agonist AM281 (Ae, $\boldsymbol{A f})$. Anti-CB1R N-Ter feeding plus (-Ter immunolabeling followed by immunoperoxidase staining is performed to maximize sensitivity. Shown are representative somas and proximal dendrites for the three conditions. The right panels in $\boldsymbol{A a}, \boldsymbol{A c}$, and $\boldsymbol{A e}$ are detailed images from the boxed areas in the left panels. Scale bars: somas, $\boldsymbol{A a}, \boldsymbol{A c}, \boldsymbol{A e}, 2 \mu \mathrm{m}$; details, $\boldsymbol{A b}, \boldsymbol{A d}, \boldsymbol{A f}, 0.5 \mu \mathrm{m}$. For the right column, the $\mathrm{S} / \mathrm{N}$ ratio of this individual cell is indicated in the lower right corner, referring to the quantification in $\boldsymbol{B}$. In control conditions $(\boldsymbol{A} \boldsymbol{a}, \boldsymbol{A} \boldsymbol{b})$, receptors are mostly found in somatic endosomes ( $\boldsymbol{A} \boldsymbol{a}$, filled arrows), with sparse labeling of the somatodendritic plasma membrane ( $\boldsymbol{A} \boldsymbol{a}$, right, empty arrow). CB1R-positive en passant axons exhibit a dense surface staining ( $\boldsymbol{A} \boldsymbol{b}$, arrowhead). Treatment with agonist WIN downregulates somatodendritic surface CB1Rs and upregulates CB1R-positive endosomes ( $\boldsymbol{A} \boldsymbol{c}$, filled arrows). Note that remaining surface (B1Rs are now found inside pits (Ad, top, empty arrow) and that axonal surface labeling is still partially present (Ad, bottom, arrowhead). Treatment with inverse agonist AM281 upregulates somatodendritic surface receptors that often appear as patches on the plasma membrane (Ae, empty arrows). Axonal labeling is not significantly different from control condition ( $\boldsymbol{A} \boldsymbol{f}$, arrowhead), as seen on (B1R-positive axons passing along CB1R-positive somas. $\boldsymbol{B}$, Quantification of CB1R translocation for endogenous (B1R (corresponding to images shown in $A$ ). $S / \mathrm{N}$ ratio represents the ratio between mean plasma membrane intensity (with eventual CB1R staining) and nuclear membrane intensity (used as control). Five neurons are quantified for each condition. WIN-treated neurons exhibit a drop in S/N ratio, showing downregulation of plasma membrane CB1Rs, whereas AM281-treated neurons exhibit higher S/N ratios, with enhanced plasma membrane labeling. The bars show the mean, the error bars indicate the SEM, and the asterisks indicate significance between the vehicle and treated conditions, as defined in Materials and Methods.

sulting in sequestration of CB1Rs on the somatic plasma membrane.

We confirmed and extended these results by using cultured hippocampal neurons transfected with FCB1-eGFP (Fig. 5A). This approach allows a more precise and sensitive measure of (1) the proportion of CB1Rs that are on the neuronal surface and (2) the translocation of CB1Rs between axonal and somatodendritic compartments of individual neurons. The first parameter measures of the ratio between fluorescence from CB1R on the plasma membrane (S; detected by rapid live staining with M1 anti-FLAG antibody) versus total CB1R fluorescence ( $\mathrm{T}$; detected with the eGFP tag), yielding the $\mathrm{S} / \mathrm{T}$ ratio (Fig. $5 B a, B b$ ). The second parameter measures the effects of pharmacological treatments on the relative compartmentalization of CB1Rs between the axon and the somatodendritic compartment. It is obtained by tracing segments along axon and dendrites (as defined morphologically) on low magnification $(20 \times)$ images of neurons and by measuring the ratio between somatodendritic and axonal staining intensities in the green (eGFP) channel, yielding the A/D ratio of the total receptor population (Fig. $5 B c$ ).

Vehicle-treated neurons show relatively few receptors present on the somatodendritic plasma membrane, accompanied by a high level of CB1R expression on their axonal arborization and numerous somatodendritic CB1R-containing endosomes (Fig. $5 A a, A b)$. The relatively low $\mathrm{A} / \mathrm{D}$ ratio (Fig. $5 B c$ ) is attributable to the very intense label of the somatodendritic endosomes accom- panied by a lower but more homogenous expression of axonal CB1Rs.

Treatment with agonist WIN55,212-2 ( $1 \mu \mathrm{M})$ for $3 \mathrm{~h}$ leads to neurons that are devoid of somatodendritic surface receptors, and CB1R-positive endosomes are abundant in the somatodendritic compartment (Fig. $5 A c, A d, B a$ ). In axons, CB1R endocytosis is also detected as a decrease in surface labeling, but seems to be rather incomplete after $3 \mathrm{~h}$ (Fig. 5Ac). This is consistent with previous data showing that disappearance of surface axonal CB1Rs is complete only after $16 \mathrm{~h}$ of agonist incubation in cultured hippocampal neurons (Coutts et al., 2001). In addition, WIN induces a significant decrease of the A/D ratio, showing that agonist-mediated activation resulted in partial translocation of the neuronal CB1R population from the axon to somatodendritic endosomes (Fig. 5Bc). Concomitant decrease of CB1R surface staining (red) and total CB1R (green) in axons suggests that the majority of CB1Rs endocytosed in the last $3 \mathrm{~h}$ from the axon have already reached the somatodendritic compartment. These results suggest that the limiting factor in agonist-induced somatodendritic translocation of axonal CB1 receptors is the relatively slow endocytosis rate from the axonal membrane but not the velocity of retrograde axonal transport.

Treatment with inverse agonist AM281 has an opposite effect on the distribution of surface CB1R: neurons exhibit a striking upregulation of CB1Rs on the somatodendritic surface after $3 \mathrm{~h}$ of treatment (Fig. $5 A e, A f, B a$ ), whereas no obvious change is de- 
tected on the axonal surface (Fig. $5 A e$ ). In the somatodendritic compartment, this phenomenon of translocation toward the plasma membrane indicates that AM281 blocks constitutive endocytosis of CB1R.

For both ligands, the extent of somatodendritic translocation ( $\mathrm{S} / \mathrm{T}$ ratio) was concentration-dependent, with significant effect from the lowest concentration tested (i.e., $200 \mathrm{~nm}$ ) (Fig. 5Bb). Namely, $200 \mathrm{~nm}$ WIN elicited significant internalization of CB1Rs from the somatodendritic plasma membrane, whereas 200 nM AM281 was able to translocate receptors from endosomes to the plasma membrane. Moreover, a concentration of $1 \mu \mathrm{M}$ AM281 was able to fully antagonize the effect of $1 \mu \mathrm{M}$ WIN, even translocating CB1Rs toward the plasma membrane as efficiently as AM281 alone. This confirms that the effect of WIN is CB1R-specific and that AM281 is a bona fide antagonist/inverse agonist of the CB1R.

If AM281 acts by stabilizing receptors in an inactive conformation, thus blocking constitutive endocytosis, its effect on CB1R distribution could be mimicked by blocking GPCR endocytosis. We thus incubated FCB1-eGFP transfected neurons with $\mathrm{MBCD}$ (5 $\mathrm{mm}$ for $30 \mathrm{~min}$ ), which strongly inhibits endocytosis, but not recycling of the transferrin receptor (Subtil et al., 1999) and of CB1R (Leterrier et al., 2004), through acute depletion of plasma membrane cholesterol. We verified that in our experimental setup, this acute treatment with MBCD significantly inhibits neuronal endocytosis as indicated by significant reduction of transferrin uptake (supplemental Fig. 3, available at www. jneurosci.org as supplemental material). After MBCD treatment, CB1Rs are upregulated on the somatodendritic surface (Fig. 5Ag,Ah) and are still present on the axonal surface (with no detectable change compared with control), suggesting that AM281 and MBCD both inhibit CB1R constitutive endocytosis in the somatodendritic compartment. Thus, receptors recycled from intracellular endosomes are accumulating on the somatodendritic plasma membrane, resulting in the observed upregulation of surface-bound receptors as measured by the rise of the S/T ratio (Fig. $5 B a$ ). Neither AM281 nor MBCD treatment significantly changed the A/D ratio of the total receptor population (Fig. $5 B c$ ) or the total number of CB1Rs, as shown by the unchanged level of total eGFP fluorescence (data not shown), indicating that their main effect in the time-scale studied (between $30 \mathrm{~min}$ and $3 \mathrm{~h}$ ) is a translocation from endosomes to plasma membrane within the somatodendritic compartment.

In conclusion, our results indicate that a constitutive cycle of $\mathrm{CB1R}$ endocytosis and recycling exist in the somatodendritic compartment of cultured neurons, similarly to the constitutive
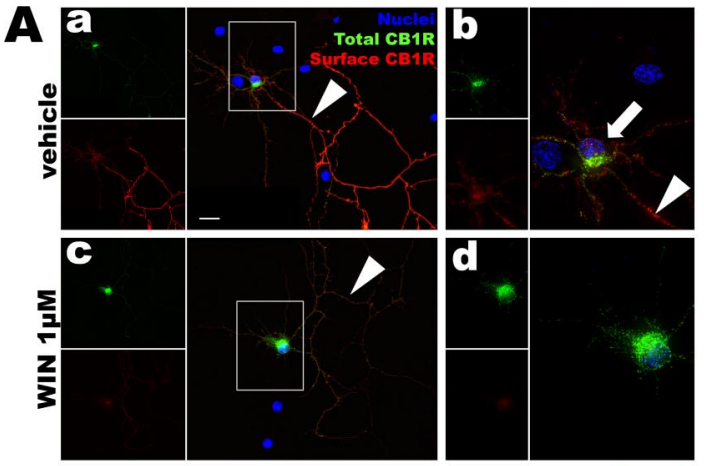

8
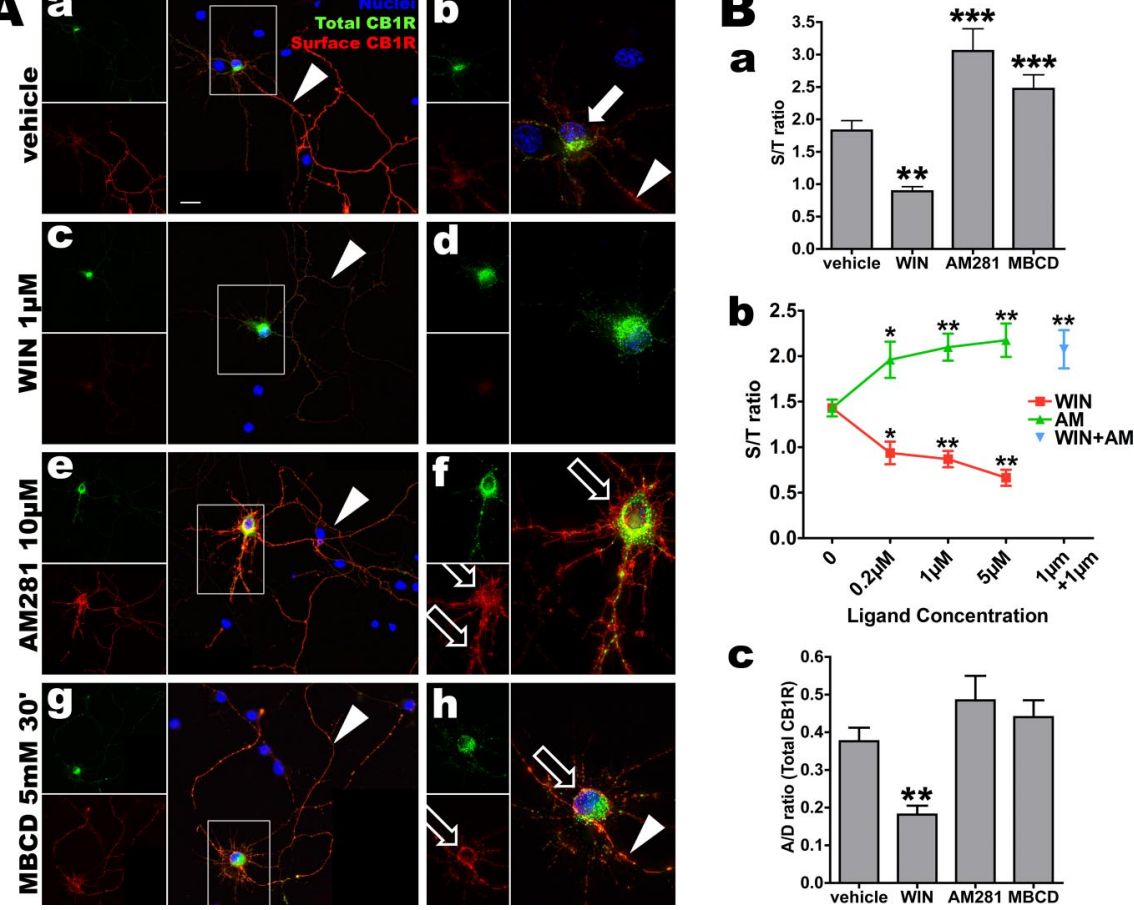

Figure 5. Somatodendritic $C B 1 R$ trafficking is dependent on the pharmacological state of $C B 1 R$. $A$, Neurons expressing FCB1eYFP are incubated for $3 \mathrm{~h}$ with vehicle (0.2\% DMSO; $\boldsymbol{A a}, \boldsymbol{A \boldsymbol { b }})$, WIN $(1 \mu \mathrm{m} ; \boldsymbol{A c}, \boldsymbol{A d}), \mathrm{AM} 281(10 \mu \mathrm{m} ; \boldsymbol{A \boldsymbol { e }}, \boldsymbol{A \boldsymbol { f }})$, or 30 min with MBCD (montage of two images) and right panels $(\boldsymbol{A} \boldsymbol{b}, \boldsymbol{A d}, \boldsymbol{A f}, \boldsymbol{A h})$ are a projection of a deconvoluted $10 \mu \mathrm{m} z$-stack $(0.2 \mu \mathrm{m} z$-step) relative staining intensities. Scale bar: (in $\mathbf{A a}) \boldsymbol{A a}-\mathbf{A h}, 20 \mu \mathrm{m}$. In control cells, surface CB1R is found on the axonal arborization $\boldsymbol{A} \boldsymbol{a}$, arrowhead) whereas somatodendritic receptors are mostly endosomal (arrow in $\boldsymbol{A} \boldsymbol{b}$, highlighting the soma of the transfected somatodendritic surface receptors are barely visible, whereas endosomes are abundant in the soma and dendrites $(\boldsymbol{A c}, \boldsymbol{A d})$. Axonal

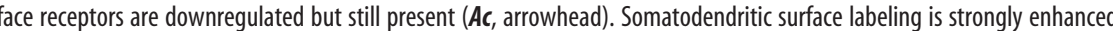
CD leads to a CB1R distribution similar to AM281 treatment with upregulation of surface expression of CB1R on the somato( rise of the S/T ratio. B $\boldsymbol{b}$, Effects of both WIN and AM281 on CB1R localization are concentration-dependent (red curve for WIN, green curve for AM281) and resulting S/T ratios are significantly different from control for all concentrations tested. AM281 ment with AM281 alone (blue data point). BC, CB1R localization in transfected neurons is quantified as the A/D ratio, that is the rio of intensities between dendrites and axon for total CB1R (green channel) in individual neurons in each condition $(n=24-26$ whereas AM281 and MBCD do not have a significant effect on the compartmentalization of CB1R between axonal and somatodendritic compartment. In all quantifications, values are mean \pm SEM and asterisks show significance between vehicle and treated conditions, as defined in Materials and Methods.

cycle characterized in HEK293 cells (Leterrier et al., 2004). This cycle is dependant on the pharmacological state of the receptor, because CB1R agonist and inverse agonist modulate equilibrium between surface and endosomal CB1R populations. Strikingly, axonal CB1Rs are localized on the plasma membrane, their spontaneous internalization is almost undetectable and even agonist treatment for $3 \mathrm{~h}$ results in a relatively slow rate of endocytosis.

Mutant proteins interfering with constitutive endocytosis of CB1Rs perturb axonal distribution of surface CB1Rs

If CB1R is constitutively endocytosed in the somatodendritic region but not in axons, continuous blockade of endocytosis could 


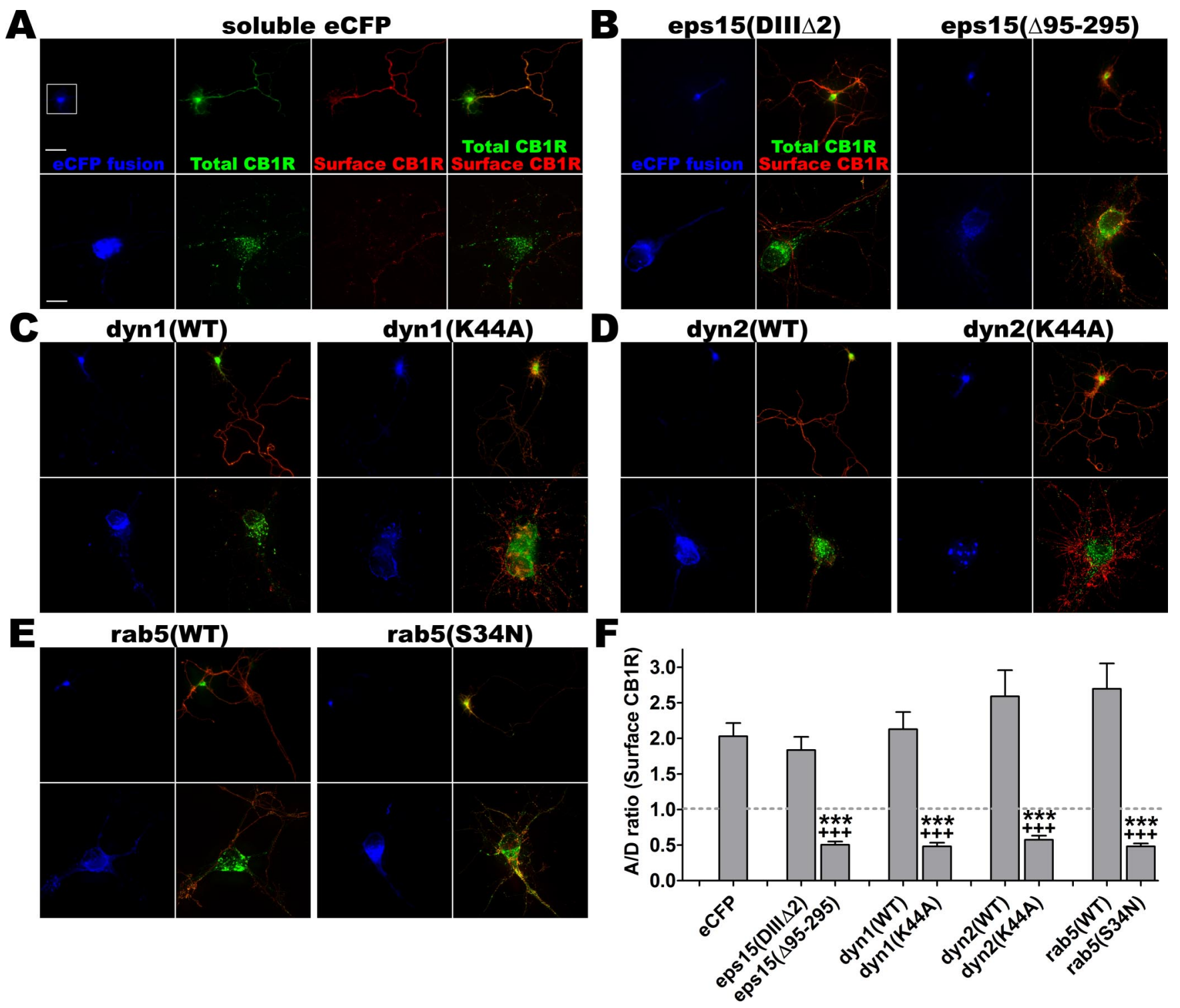

Figure 6. Inhibition of neuronal endocytosis shifts surface expression of (B1R from the axon to soma and dendrites. Hippocampal neurons at DIV 8 are cotransfected with FCB1-eYFP (green) and a fusion of eCFP (blue) with various proteins: soluble eCFP as a control (A), inactive mutant eps15(DIII $\Delta 2$ ) or dominant-negative eps15( $\Delta 95-295)$ (B), wild-type dyn1(WT) or dominant-negative dyn1(K44A) (C), wild-type dyn2(WT) or dominant-negative dyn2(K44A) (D), wild-type rab5(WT) or dominant-negative rab5(S34N) (E). Twenty-four hours later, they are stained for surface CB1Rs (red) and fixed. For each condition, the top panel is a $20 \times$ image of the neuron with its axonal arborization (scale bar, $50 \mu \mathrm{m}$ ) and the bottom panel is a z-projection of a deconvoluted $10 \mu \mathrm{m}$ stack $(0.2 \mu \mathrm{m}$ Z-step) taken with a $100 \times$ objective (scale bar, $10 \mu \mathrm{m})$. Wild-type or inactive proteins do not change polarization of CB1R surface expression (red labeling, left panels), whereas dominant-negative mutants of eps15 (B), dyn1 (C), dyn2 (D), and rab5 (E) strongly upregulate somatodendritic surface (B1R expression (red somatodendritic labeling, right panels). $\boldsymbol{F}$, Quantification of the $A / D$ ratio, that is the ratio of intensities between dendrites and axon for surface CB1R (red channel) in individual neurons in each condition ( $n=20$ neurons from two independent experiments). Values are mean \pm SEM and asterisks show the significance between eCFP alone and eCFP-protein fusions. Crosses show significance between control eCFP-protein fusion (inactive mutant for eps 15 and WT proteins for rab5 and dynamins) and dominant-negative eCFP-protein fusion. Quantifications show that eCFP alone or control proteins (either WT or inactive mutant) do not significantly modify the axonal surface CB1R distribution $(A / D>1)$, but dominant-negative mutants reverse the polarity of CB1R surface expression, which is then mostly somatodendritic $(A / D<1)$, showing the role of endocytosis in proper surface distribution of $C B 1 R$ in neurons.

perturb the polarized distribution of surface CB1Rs. Thus, we coexpressed CB1R with different wild-type or dominant-negative mutant proteins, known to inhibit endocytosis, and examined the effects on the subcellular distribution of CB1R. The effect of the cotransfected proteins on the axonal polarization of surface CB1Rs was assessed by the $\mathrm{A} / \mathrm{D}$ ratio measured on the surface $\mathrm{CB} 1 \mathrm{R}$ images (red channel) for neurons fixed and stained $24 \mathrm{~h}$ after cotransfection of FCB1-eYFP with various wild-type or mutant proteins.

We studied the effects of dominant-negative mutants of eps15, dynamin-1, dynamin-2, and rab5 (Fig. 6). Eps15 is an AP-2 binding protein that is involved in clathrin-mediated endocytosis (Benmerah et al., 2000). Dynamin GTPases participate in the last step of endocytosis by pinching the vesicle from the plasma membrane (Cao et al., 1998). Finally, early steps in CB1R endocytic trafficking are driven by the small GTPase rab5 (Leterrier et al., 2004), a protein that has been shown to participate in endocytosis of numerous GPCRs (Seachrist and Ferguson, 2003). Coexpression of the eCFP-tagged control constructs do not significantly change the distribution of CB1R compared with soluble eCFP (Fig. 6A, $B-E$, left). Strikingly, cotransfection of the dominantnegative mutant proteins eps15( $\Delta 95-295)$ as well as dyn $1 \mathrm{~K}(44 \mathrm{~A})$, dyn2(K44A), and GDP-locked rab5(S34N) enhances surface expression of CB1R on the somatodendritic membrane with a concomitant decrease of CB1R expression at the axonal surface (Fig. 
$6 B-E$, right), leading to a more somatodendritic phenotype and a fall of the A/D ratio (Fig. $6 F$ ).

These results suggest that in neurons, CB1Rs are constitutively endocytosed in an eps15- and dynamin-dependent manner and that subsequent trafficking depends on rab5. Moreover, inhibition of this constitutive endocytosis retains CB1Rs on the somatodendritic plasma membrane and leads to improper distribution of surface-localized CB1Rs, showing that constitutive endocytosis from the somatodendritic plasma membrane is necessary for the polarized distribution of CB1R in neurons.

Kinetic analysis unravels the role of constitutive activity and endocytosis in axonal targeting of CB1Rs

Whereas CB1R constitutively cycles between cell surface and intracellular endosomes in the somatodendritic region, the situation is clearly different in the axonal compartment. Axonal CB1Rs are localized on the surface at steady state and their endocytosis is slow even in the presence of an agonist (our results above) (Coutts et al., 2001). Interestingly, this difference in endocytic rates may be sufficient to explain the polarized distribution of CB1Rs on the neuronal surface at steady state. In this scenario, newly synthesized CB1Rs are first delivered indiscriminately to the somatodendritic and the axonal plasma membrane. Subsequently, distribution polarized to the axonal surface is established and maintained by the difference between the spontaneous endocytosis rates from the somatodendritic and axonal plasma membrane. To test this hypothesis, we followed the kinetics of the insertion of CB1R into the plasma membrane by adapting a BFA-release protocol (Wisco et al., 2003; Fache et al., 2004). BFA reversibly inhibits Arf1-dependent intracellular transport, blocking export of membrane proteins that accumulate in a mixed ER/golgi compartment. After washout of BFA, protein trafficking resumes in a synchronized manner, inducing a "pulse" of protein export and targeting to their functional subcellular compartments.

Neurons were thus transfected with FCB1-eGFP for $3 \mathrm{~h}$, treated overnight with BFA $(0.5-0.75 \mu \mathrm{g} / \mathrm{ml})$, then allowed to resume protein trafficking by washing out BFA in the presence of vehicle or inverse agonist AM281 $(10 \mu \mathrm{M})$, and fixed at different times after washout (Fig. 7A). Live staining of surface receptors was performed by rapid incubation with anti-FLAG antibody preceding fixation and staining with secondary antibody. Transfected neurons were then systematically searched and classified according to the distribution of surface CB1R (supplemental Fig. 4 , available at www.jneurosci.org as supplemental material) (see Materials and Methods).

In control cells with no BFA block, $78 \%$ of transfected neurons exhibit axonal distribution of CB1R (Fig. $7 B$ ). Immediately after BFA washout, all neurons treated with BFA overnight exhibit intracellular accumulation of CB1Rs. Strikingly, between 4 and $8 \mathrm{~h}$ after BFA washout, a significant proportion of neurons is observed exhibiting uniform (35\%) and somatodendritic surface staining (23\%). Twenty-four hours after washout, CB1R surface distribution approaches the distribution observed in untreated neurons, with a majority of neurons displaying a predominantly axonal distribution $(67 \%)$. This transient appearance of CB1Rs on the somatodendritic surface suggests that CB1Rs are not directly targeted to axons but are rather nonspecifically sent toward the entire neuronal plasma membrane. Thus, most of them appear first on the somatodendritic membrane from where they are subsequently removed by constitutive endocytosis, whereas receptors reaching the axonal surface are maintained on the plasma membrane. Accordingly, if the BFA washout is followed by incu-
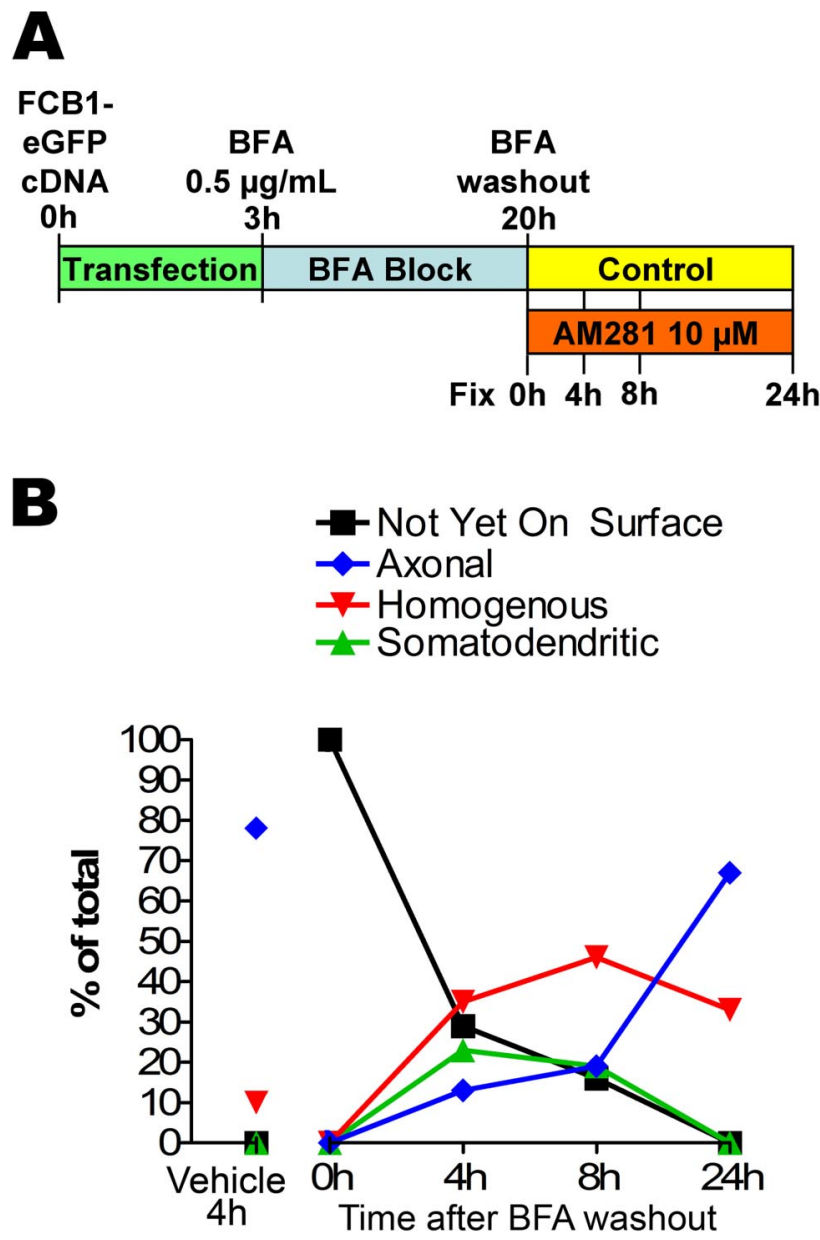

C

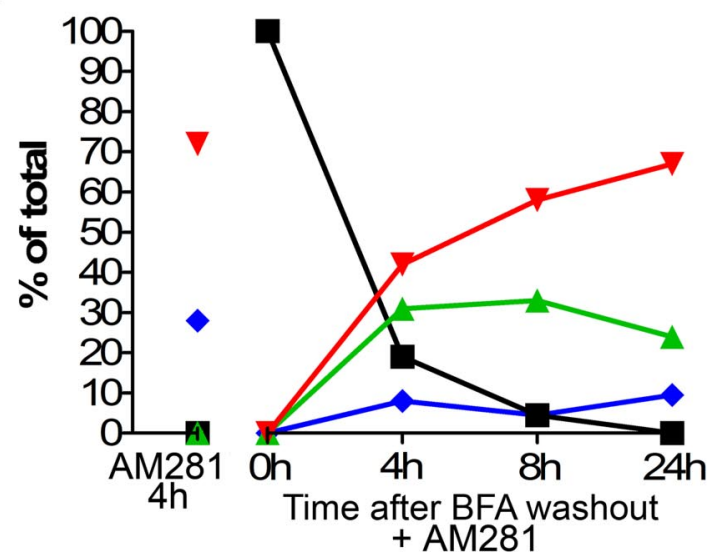

Figure 7. Time course of CB1R insertion in the neuronal plasma membrane. $\boldsymbol{A}$, Diagram of the time course experiment. DIV 8 neurons are transfected with FCB1-eGFP by 3 h incubation in transfection medium. They are then incubated overnight with $0.5-0.75 \mu \mathrm{g} / \mathrm{ml}$ BFA to block protein export from the golgi. After extensive washes (BFA washout), neurons are incubated in either regular medium or in the presence or $10 \mu \mathrm{MAM} 281$ for $0,4,8$, and $24 \mathrm{~h}$. At these time points, they are labeled for surface CB1Rs and fixed. The proportion of each category is determined for each condition and time point ( $n=50-150$ neurons in each of two independent experiments leading to comparable results). $\boldsymbol{B}$, Time course of $C B 1$ R surface targeting in control conditions. Just after BFA washout ( $0 \mathrm{~h})$, no $C B 1 \mathrm{R}$ is present at the neuronal plasma membrane. Then, a transient appearance of somatodendritic surface CB1Rs (at 4 and $8 \mathrm{~h}$ ) precedes establishment of the proper axonal distribution (after $24 \mathrm{~h}$ ). This shows that $C B 1 R$ is likely to be first inserted in the somatodendritic plasma membrane before being endocytosed and sent to the axon. C, Time course of CB1R surface targeting in the presence of $10 \mu \mathrm{mAM} 281$. Interestingly, the inverse agonist sequesters CB1Rs that transit on the somatodendritic plasma membrane, thus inhibiting emergence of the axonal surface distribution of CB1R (majority of "somatodendritic" and "uniform" phenotypes $24 \mathrm{~h}$ after BFA washout in the presence of AM281). 
bation with inverse agonist AM281, the final surface distribution of CB1R is reversed (Fig. 7C). Although CB1R appears $4 \mathrm{~h}$ after washout on the somatodendritic surface, subsequent endocytic elimination is impaired, leading to neurons displaying mostly uniform and somatodendritic distributions $24 \mathrm{~h}$ after washout (67 and 24\%, respectively). These results show that most CB1Rs transiently appear on the somatodendritic plasma membrane before reaching the axon and that inverse agonist AM281 blocks this mechanism by sequestering CB1Rs on the somatodendritic plasma membrane. Thus, constitutive activity is the driving force of CB1R elimination from the somatodendritic plasma membrane and is necessary for the establishment of axonal polarity observed for surface CB1Rs.

\section{Discussion}

We have investigated spontaneous and ligand-induced intracellular translocation of CB1R in cultured hippocampal neurons to examine how an important property of this receptor, constitutive activity, might influence receptor function through regulation of its subneuronal localization. Our results indicate that neuronal CB1Rs are constitutively activated in the somatodendritic compartment of neurons. This constitutive activity results in a permanent cycle of endocytosis and recycling, leading to a predominantly intracellular localization in the somatodendritic compartment at steady state. Pharmacological or molecular blockade of this somatodendritic cycle disrupts axonal polarization of CB1R. Thus, the somatodendritic, constitutive activitydriven endosomal pathway supplies axons with CB1Rs and provides a unique mechanistic link between receptor pharmacology and functional targeting.

\section{Somatodendritic cycle of endocytosis and recycling}

We have previously described that in non-neuronal cells, constitutive activity of CB1Rs results in a permanent cycle of endocytosis and recycling (Leterrier et al., 2004). A growing body of evidence indicates that this behavior is likely a general phenomenon for constitutively active GPCRs (Whistler et al., 2002; Fourgeaud et al., 2003; Leterrier et al., 2004; Marion et al., 2004; Morris et al., 2004). However, these results were obtained in heterologous expression systems, so the physiological relevance of this phenomenon remains unknown. To our knowledge, no physiological role has been proposed for this cycling either. Our present data suggests that constitutive endocytosis and recycling between plasma membrane and endosomes is relevant to describe the behavior of endogenous CB1Rs in the somatodendritic compartment of neurons. In our study, two experimental results show the constitutive endocytosis of CB1Rs in neurons. First, in the somatodendritic compartment, CB1Rs are present in intracellular vesicles as also reported previously (Katona et al., 1999; Coutts et al., 2001). Second, these endosomal receptors have an endocytic origin as shown by the antibody-feeding experiments.

Constitutive somatodendritic endocytosis of CB1R was found to follow the clathrin-dependent endocytosis pathway, similarly to endocytosis of other neuronal GPCRs like neurokinin NK1 receptor (Schmidlin et al., 2001), but not mGluR5 (Fourgeaud et al., 2003). Indeed, endocytosed CB1Rs colocalized extensively with cotransfected clathrin. Moreover, inhibition of clathrinmediated endocytosis by acute depletion of plasma membrane cholesterol (MBCD), as well as cotransfection with dominantnegative mutants of eps15, dynamin1, dynamin2, and rab5, impaired spontaneous endocytosis of CB1Rs, as indicated by its upregulation on the somatodendritic plasma membrane.

Importantly, inverse agonist AM281 also interrupts spontane- ous CB1R endocytosis by sequestering both transfected and endogenous receptors on the somatodendritic plasma membrane. If we interpret inverse agonist action as stabilization of an inactive conformation of the receptor (Milligan and Bond, 1997), this is as a strong indication that CB1Rs are constitutively activated in the somatodendritic compartment of neurons. The source of constitutive activation for somatodendritic CB1Rs could result from the well documented intrinsic constitutive activity of CB1R (Bouaboula et al., 1997; Pan et al., 1998; Rinaldi-Carmona et al., 1998) but also from endocannabinoids present in the culture medium. However, control experiments measuring agonistinduced endocytosis of CB1-eGFP in HEK293 cells incubated with supernatant medium from neuronal cultures did not detect significant endocannabinoid-induced CB1R endocytosis (unpublished results), in line with the results of Pan et al. (1998) who did not detect endocannabinoid effects on calcium currents in dorsal root ganglia neuronal cultures. Formal exclusion of endocannabinoid effects is difficult though, because of the lack of proven neutral antagonists for the CB1R (i.e., ligands that antagonize agonist binding without influencing receptor conformational equilibrium).

The last step in the constitutive cycle involves constitutive recycling of $\mathrm{CB} 1 \mathrm{R}$ from endosomes to the plasma membrane. The effects of MBCD and AM281 suggest that there is a constant supply of CB1Rs that bring receptors to the plasma membrane allowing upregulation of the surface population, via inhibition of $\mathrm{CB} 1 \mathrm{R}$ endocytosis. As the effects take place after short-term treatment ( $30 \mathrm{~min}$ and $3 \mathrm{~h}$, respectively), these receptors are unlikely to be neosynthesized receptors but rather recycled receptors, like is the case in HEK293 cells (Leterrier et al., 2004).

\section{In axons of differentiated neurons, CB1Rs accumulate on the plasma membrane}

Strikingly, the axonal CB1R population behaves quite differently. Our observations using live-cell surface immunolabeling followed by fluorescent optical or electron microscopy confirm previous findings (Coutts et al., 2001), showing that expression of CB1Rs gradually increases on the axonal surface as neurons mature and diminishes slowly even after prolonged agonist treatment. Agonist-induced endocytosis of surface CB1Rs is complete after $16 \mathrm{~h}$ (Coutts et al., 2001), and axonal surface CB1Rs are not upregulated after inverse agonist treatment. On the contrary, in the somatodendritic compartment, our data show that CB1R endocytosis is rapid and complete after $3 \mathrm{~h}$, similarly to other cell types like HEK293 cells (Leterrier et al., 2004) or other heterologous expression systems (Rinaldi-Carmona et al., 1998; Hsieh et al., 1999). This suggests the presence of a less effective endocytic machinery for CB1Rs in axons compared with the somatodendritic compartment. This slow rate of endocytosis could constitute the limiting factor that induces sequestration of CB1Rs on the plasma membrane and is in fact supported by description of differential endocytic pathways in somatodendritic and axonal compartments (Mundigl et al., 1993). A detailed EM study by Parton et al. (1992) showed that in axons, early endosomes were found only in presynaptic terminals and in varicosities but not in the axonal shaft, suggesting that axonal endocytosis is restricted to the presynaptic sites. Indeed, these authors have shown that internalization of fluid-phase markers was significantly reduced in mature axons, as compared with immature axons or to the somatodendritic membrane. Thus, CB1R traffic proceeds differently in the axonal and somatodendritic compartments, with retention at the axonal plasma membrane and selective elimination 
through constitutive endocytosis from the somatodendritic surface.

\section{Axonal targeting trough constitutive activity-dependent somatodendritic endocytosis}

The above considerations lead us to propose a simple mechanism of CB1R targeting based on its constitutive activity and the difference between endocytosis rates in the axonal and somatodendritic compartments. Newly synthesized receptors are delivered to both neuronal compartments, followed by elimination from the somatodendritic membrane because of constitutive activitydependent endocytosis. The same constitutive activity leads to significantly reduced endocytosis from the axonal membrane, because of the lower endocytic capacity of mature axons. Multiple cycles of endocytosis and recycling thus lead to a gradual sequestration of receptors on the axonal plasma membrane. This model (supplemental Fig. 5, available at www.jneurosci.org as supplemental material) requires two well segregated membrane compartments, which is likely the case in neurons, where a diffusion barrier at the axonal initial segment prevents axonal and somatodendritic membrane proteins to mix (Winckler et al., 1999; Nakada et al., 2003). This model is supported by our kinetic data obtained using time-resolved analysis of CB1R distribution after BFA washout, a procedure that facilitates the observation of postgolgi protein transport. First, newly synthesized receptors accumulate in the first hours on the somatodendritic plasma membrane. Second, significant axonal polarization of CB1Rs is only achieved after $24 \mathrm{~h}$. Third, blockade of constitutive activitydependent somatodendritic endocytosis of CB1R by inverse agonist AM281 also prevents proper axonal polarization of surface $\mathrm{CB} 1 \mathrm{Rs}$, leading to a nonpolarized distribution or even to reversal of the polarized distribution of surface CB1Rs. Interestingly, this model also implies that a substantial proportion of CB1Rs expressed on the axonal plasma membrane have been transcytosed from the somatodendritic plasma membrane.

Selective somatodendritic elimination, together with axonal retention as a targeting mechanism, was recently proposed for a chimera protein consisting of the cytoplasmic tail of the axonal sodium channel Nav1.2 fused to a reporter protein (Garrido et al., 2001), as well as for VAMP2 (vesicle-associated membrane protein 2), a synaptic vesicle V-SNARE (soluble $N$-ethylmaleimide-sensitive factor attachment protein receptor) (Sampo et al., 2003), and NgCAM (neuron-glia cell adhesion molecule) (Wisco et al., 2003). Despite important common characteristics, there is a fundamental difference between these targeting models and the model we propose for CB1R: the aforementioned studies identified static intracellular motifs recognized by the somatodendritic endocytic machinery, whereas CB1R endocytosis is caused by reversible adoption of an active conformation by the receptor. In other words, the axonal targeting information in the above models is encoded in the primary structure of the proteins, whereas for CB1R it is coded in the tertiary structure.

In conclusion, in this report we (1) show the implication of compartment-selective endocytosis in the axonal targeting of CB1R, (2) identify intracellular pathways involved in neuronal endocytosis of CB1R, (3) propose a general mechanism of axonal retention, the reduced endocytic capacity of mature axons, and (4) perhaps most importantly, show that for the GPCR CB1R, the driving force of axonal targeting is the pharmacologically active state of the receptor. The validation of the constitutive activitydependent axonal targeting cycle for axonal receptors other than $\mathrm{CB} 1 \mathrm{R}$ would possibly enhance our understanding of the physiology and physiopathology for several therapeutically important neurotransmitter systems, and could also provide an additional pharmacodynamical framework (i.e., interference with axonal targeting) to interpret the chronic effects of inverse agonist drugs.

\section{References}

Benmerah A, Poupon V, Cerf-Bensussan N, Dautry-Varsat A (2000) Mapping of Eps15 domains involved in its targeting to clathrin-coated pits. J Biol Chem 275:3288-3295.

Bouaboula M, Perrachon S, Milligan L, Canat X, Rinaldi-Carmona M, Portier M, Barth F, Calandra B, Pecceu F, Lupker J, Maffrand JP, Le Fur G, Casellas P (1997) A selective inverse agonist for central cannabinoid receptor inhibits mitogen-activated protein kinase activation stimulated by insulin or insulin-like growth factor 1. Evidence for a new model of receptor/ligand interactions. J Biol Chem 272:22330-22339.

Cao H, Garcia F, McNiven MA (1998) Differential distribution of dynamin isoforms in mammalian cells. Mol Biol Cell 9:2595-2609.

Coutts AA, Anavi-Goffer S, Ross RA, MacEwan DJ, Mackie K, Pertwee RG, Irving AJ (2001) Agonist-induced internalization and trafficking of cannabinoid CB1 receptors in hippocampal neurons. J Neurosci 21:2425-2433.

de Ligt RA, Kourounakis AP, Ijzerman AP (2000) Inverse agonism at G-protein-coupled receptors: (patho)physiological relevance and implications for drug discovery. Br J Pharmacol 130:1-12.

Dotti CG, Sullivan CA, Banker GA (1988) The establishment of polarity by hippocampal neurons in culture. J Neurosci 8:1454-1468.

Engqvist-Goldstein AE, Warren RA, Kessels MM, Keen JH, Heuser J, Drubin DG (2001) The actin-binding protein Hip1R associates with clathrin during early stages of endocytosis and promotes clathrin assembly in vitro. J Cell Biol 154:1209-1223.

Fache MP, Moussif A, Fernandes F, Giraud P, Garrido JJ, Dargent B (2004) Endocytotic elimination and domain-selective tethering constitute a potential mechanism of protein segregation at the axonal initial segment. J Cell Biol 166:571-578.

Fletcher TL, De Camilli P, Banker G (1994) Synaptogenesis in hippocampal cultures: evidence indicating that axons and dendrites become competent to form synapses at different stages of neuronal development. J Neurosci 14:6695-6706.

Fourgeaud L, Bessis AS, Rossignol F, Pin JP, Olivo-Marin JC, Hemar A (2003) The metabotropic glutamate receptor mGluR5 is endocytosed by a clathrin-independent pathway. J Biol Chem 278:12222-12230.

Garrido JJ, Fernandes F, Giraud P, Mouret I, Pasqualini E, Fache MP, Jullien F, Dargent B (2001) Identification of an axonal determinant in the C-terminus of the sodium channel Na(v)1.2. EMBO J 20:5950-5961.

Gifford AN, Tang Y, Gatley SJ, Volkow ND, Lan R, Makriyannis A (1997) Effect of the cannabinoid receptor SPECT agent, AM 281, on hippocampal acetylcholine release from rat brain slices. Neurosci Lett 238:84-86.

Goslin K, Banker G (1990) Rapid changes in the distribution of GAP-43 correlate with the expression of neuronal polarity during normal development and under experimental conditions. J Cell Biol 110:1319-1331.

Goslin K, Asmuyssen H, Banker G (1998) Rat hippocampal neurons in lowdensity cultures. In: Culturing nerve cells, Ed 2 (Banker G, Goslin K, eds), pp 339-370. Cambridge, MA: MIT.

Guan XM, Kobilka TS, Kobilka BK (1992) Enhancement of membrane insertion and function in a type IIIb membrane protein following introduction of a cleavable signal peptide. J Biol Chem 267:21995-21998.

Howlett AC, Barth F, Bonner TI, Cabral G, Casellas P, Devane WA, Felder CC, Herkenham M, Mackie K, Martin BR, Mechoulam R, Pertwee RG (2002) International union of pharmacology. XXVII. Classification of cannabinoid receptors. Pharmacol Rev 54:161-202.

Hsieh C, Brown S, Derleth C, Mackie K (1999) Internalization and recycling of the CB1 cannabinoid receptor. J Neurochem 73:493-501.

Jolimay N, Franck L, Langlois X, Hamon M, Darmon M (2000) Dominant role of the cytosolic C-terminal domain of the rat $5-\mathrm{HT}_{1 \mathrm{~B}}$ receptor in axonal-apical targeting. J Neurosci 20:9111-9118.

Katona I, Sperlagh B, Sik A, Kafalvi A, Vizi ES, Mackie K, Freund TF (1999) Presynaptically located CB1 cannabinoid receptors regulate GABA release from axon terminals of specific hippocampal interneurons. J Neurosci 19:4544-4558.

Kenakin T (2004) Efficacy as a vector: the relative prevalence and paucity of inverse agonism. Mol Pharmacol 65:2-11.

Leterrier C, Bonnard D, Carrel D, Rossier J, Lenkei Z (2004) Constitutive 
endocytic cycle of the CB1 cannabinoid receptor. J Biol Chem 279:36013-36021.

Marion S, Weiner DM, Caron MG (2004) RNA editing induces variation in desensitization and trafficking of 5-hydroxytryptamine $2 \mathrm{c}$ receptor isoforms. J Biol Chem 279:2945-2954.

Matsuda LA, Lolait SJ, Brownstein MJ, Young AC, Bonner TI (1990) Structure of a cannabinoid receptor and functional expression of the cloned cDNA. Nature 346:561-564.

Meijering E, Jacob M, Sarria JC, Steiner P, Hirling H, Unser M (2004) Design and validation of a tool for neurite tracing and analysis in fluorescence microscopy images. Cytometry A 58:167-176.

Milligan G (2003) Constitutive activity and inverse agonists of G-proteincoupled receptors: a current perspective. Mol Pharmacol 64:1271-1276.

Milligan G, Bond RA (1997) Inverse agonism and the regulation of receptor number. Trends Pharmacol Sci 18:468-474.

Miserey-Lenkei S, Parnot C, Bardin S, Corvol P, Clauser E (2002) Constitutive internalization of constitutively active angiotensin II AT(1A) receptor mutants is blocked by inverse agonists. J Biol Chem 277:5891-5901.

Morisset S, Rouleau A, Ligneau X, Gbahou F, Tardivel-Lacombe J, Stark H, Schunack W, Ganellin CR, Schwartz JC, Arrang JM (2000) High constitutive activity of native $\mathrm{H}_{3}$ receptors regulates histamine neurons in brain. Nature 408:860-864.

Morris DP, Price RR, Smith MP, Lei B, Schwinn DA (2004) Cellular trafficking of human $\alpha 1 \mathrm{a}$-adrenergic receptors is continuous and primarily agonist-independent. Mol Pharmacol 66:843-854.

Mundigl O, Matteoli M, Daniell L, Thomas-Reetz A, Metcalf A, Jahn R, De Camilli P (1993) Synaptic vesicle proteins and early endosomes in cultured hippocampal neurons: differential effects of Brefeldin A in axon and dendrites. J Cell Biol 122:1207-1221.

Nakada C, Ritchie K, Oba Y, Nakamura M, Hotta Y, Iino R, Kasai RS, Yamaguchi K, Fujiwara T, Kusumi A (2003) Accumulation of anchored proteins forms membrane diffusion barriers during neuronal polarization. Nat Cell Biol 5:626-632.

Nyiri G, Cserep C, Szabadits E, Mackie K, Freund TF (2005) CB1 cannabinoid receptors are enriched in the perisynaptic annulus and on pretermi- nal segments of hippocampal GABAergic axons. Neuroscience 136:811-822.

Pan X, Ikeda SR, Lewis DL (1998) SR 141716A acts as an inverse agonist to increase neuronal voltage-dependent $\mathrm{Ca}^{2+}$ currents by reversal of tonic CB1 cannabinoid receptor activity. Mol Pharmacol 54:1064-1072.

Parton RG, Simons K, Dotti CG (1992) Axonal and dendritic endocytic pathways in cultured neurons. J Cell Biol 119:123-137.

Pertwee RG (2005) Inverse agonism and neutral antagonism at cannabinoid CB1 receptors. Life Sci 76:1307-1324.

Pierce KL, Premont RT, Lefkowitz RJ (2002) Seven-transmembrane receptors. Nat Rev Mol Cell Biol 3:639-650.

Rinaldi-Carmona M, Le Duigou A, Oustric D, Barth F, Bouaboula M, Carayon P, Casellas P, Le Fur G (1998) Modulation of CB1 cannabinoid receptor functions after a long-term exposure to agonist or inverse agonist in the Chinese hamster ovary cell expression system. J Pharmacol Exp Ther 287:1038-1047.

Sampo B, Kaech S, Kunz S, Banker G (2003) Two distinct mechanisms target membrane proteins to the axonal surface. Neuron 37:611-624.

Schmidlin F, Dery O, DeFea KO, Slice L, Patierno S, Sternini C, Grady EF, Bunnett NW (2001) Dynamin and Rab5a-dependent trafficking and signaling of the neurokinin 1 receptor. J Biol Chem 276:25427-25437.

Seachrist JL, Ferguson SS (2003) Regulation of G-protein-coupled receptor endocytosis and trafficking by Rab GTPases. Life Sci 74:225-235.

Subtil A, Gaidarov I, Kobylarz K, Lampson MA, Keen JH, McGraw TE (1999) Acute cholesterol depletion inhibits clathrin-coated pit budding. Proc Natl Acad Sci USA 96:6775-6780.

Whistler JL, Gerber BO, Meng EC, Baranski TJ, von Zastrow M, Bourne HR (2002) Constitutive activation and endocytosis of the complement factor 5a receptor: evidence for multiple activated conformations of a G-protein-coupled receptor. Traffic 3:866-877.

Winckler B, Forscher P, Mellman I (1999) A diffusion barrier maintains distribution of membrane proteins in polarized neurons. Nature 397:698-701.

Wisco D, Anderson ED, Chang MC, Norden C, Boiko T, Folsch H, Winckler B (2003) Uncovering multiple axonal targeting pathways in hippocampal neurons. J Cell Biol 162:1317-1328. 